\title{
Basal lamina structural alterations in human asymmetric aneurismatic aorta
}

\author{
M. Cotrufo, ${ }^{*}$ L. De Santo, ${ }^{*}$ A. Della Corte, ${ }^{*}$ F. Di Meglio, ${ }^{\circ}$ G. Guerra, ${ }^{\circ}$ C. Quarto, ${ }^{*}$ S. Vitale, ${ }^{\circ}$ \\ C. Castaldo, ${ }^{\circ}$ S. Montagnani ${ }^{\circ}$
}

*Department of Cardio-Thoracic and Respiratory Sciences, Second University of Naples, V Monaldi Hospital; ${ }^{\circ}$ Department of Biomorphological and Functional Sciences, Federico II University, Naples, Italy

(C)2005, European Journal of Histochemistry

Basal lamina (BL) is a crucial mechanical and functional component of blood vessels, constituting a sensor of extracellular microenvironment for endothelial cells and pericytes. Recently, an abnormality in the process of matrix microfibrillar component remodeling has been advocated as a mechanism involved in the development of aortic dilation. We focused our attention on BL composition and organization and studied some of the main components of the Extracellular Matrix such as Tenascin, Laminins, Fibronectin, type I, III and IV Collagens. We used surgical fragments from 27 patients, submitted to operation because of aortic root aneurysm and 5 normal aortic wall specimens from heart donors without any evidence for aneurysmal or atherosclerotic diseases of the aorta. Two samples of aortic wall were harvested from each patient, proximal to the sinotubular junction at the aortic convexity and concavity. Each specimen was processed both for immunohistochemical examination and molecular biology study. We compared the convexity of each aortic sample with the concavity of the same vessel, and both of them with the control samples. The synthesis of mRNA and the levels of each protein were assessed, respectively, by RTPCR and Western Blot analysis. Immunohistochemistry elucidated the organization of BL, whose composition was revealed by molecular biology. All pathological samples showed a wall thinner than normal ones. Basal lamina of the aortic wall evidentiated important changes in the tridimensional arrangement of its major components which lost their regular arrangement in pathological specimens. Collagen I, Laminin $\alpha 2$ chain and Fibronectin amounts decreased in pathological samples, while type IV Collagen and Tenascin synthesis increased. Consistently with the common macroscopic observation that ascending aorta dilations tend to expand asymmetrically, with prevalent involvement of the vessel convexity and relative sparing of the concavity, Collagen type IV is more evident in the concavity and Tenascin in the convexity.

Key words: extracellular matrix, aortic dilation, basal lamina.

Correspondence: S. Montagnani,

Sezione di Anatomia Umana e Istologia,

Dipartimento di Scienze Biomorfologiche e Funzionali,

Via Pansini 5, 80131, Naples, Italy.

Tel: +39.081.7463419.

Fax: +39.081 .5469803$

E-mail: montagna@unina.it

Paper accepted on July 28, 2004

European Journal of Histochemistry

2005; vol. 49 issue 3 (Oct-Dec): 363-370
$\mathrm{B}$ asal Lamina ( $B L)$ is a thin sheet of highly specialized extracellular matrix which is present at the epithelial/mesenchymal interface of most tissues. Originally believed to serve as a selective barrier and scaffold to which cells adhere, it has become evident that individual components of the $\mathrm{BL}$ influence biological activities such as cell growth, differentiation and migration. Thus, BL has been recognized as an important regulator of cell behaviour, rather than just a structural feature of tissues. It is also a crucial mechanical and functional component of blood vessels, constituting a sensor of extracellular microenvironment for endothelial cells and pericytes.

$\mathrm{BL}$ has significant tensile strenght and provides physical support for structures such as renal tubules, blood vessels and lens capsule. During vascular contraction, BL surrounding smooth muscle has to be flexible enough to accomodate drastic changes in shape. Perhaps the polygonal network of type IV Collagen provides this plasticity. Type IV Collagen is one of the main components of $B L$ and is the most abundant constituent of the vascular $\mathrm{BL}$. It is also termed network-forming Collagen due to its capacity to self-assemble into organized network, which is crucial for BL integrity, stability and structural organization. Laminins are BL glycoproteins which influence cell adhesion, spreading and migration. They regulate cell migration, proliferation and differentiation during embryonic development, as well as in the adult life and in some pathological conditions. Laminins are heterotrimers formed by three chains $(\alpha, \beta$ and $\gamma)$; they interact with cells through integrins, dystroglycan and other receptors, so contributing to the maintenance of tissue phenotype and survival. Laminin $2(\alpha 2 \beta 1 \gamma 1)$ and Laminin $4(\alpha 2 \beta 2 \gamma 1)$ which are also called merosins, Laminin $8(\alpha 2 \beta 2 \gamma 1)$ and Laminin 10 $(\alpha 5 \beta 1 \gamma 1)$ are characteristically present in cardiac, skeletal and smooth muscle and in human vessels (Miner, 2004). 
Recent studies on animal experimental models demonstrated that Fibronectin and Tenascin overexpression is related to arterial remodeling processes in response to mechanical factors such as the hypertensive stimulus (Mackie, 1992; Bardy, 1996). In the human aorta, Fibronectin has been found as a component of basal lamina-like layers connecting smooth muscle cells to each other as well as to oxytalan fibers (Dingemans, 2000).

Recently, the normal aortic root and valve have been documented to constitute an asymmetric, highly complex structure (Grande, 1998; Choo, 1999; Dagum, 1999; Lansac, 2002) in which hemodynamic stresses are not equally distributed along the circumference of the vessel. It is well known that Medial Degeneration (MD) changes underlie the ageing process of the normal aorta (Schlatmann, 1977), and this has been attributed to hemodynamically induced biomechanical fatigue caused by longitudinal stretching and circumferential extension effects of the pulse waves during life (Stehbens, 1996; Robicsek, 2003). This evidence has strongly contributed to the development of the hemodynamic theory to explain the genesis of MD. Consistently with the common macroscopic observation that ascending aorta dilations tend to expand asymmetrically with prevalent involvement of the convex aspect of the vessel and relative sparing of the concavity (Bauer, 2002; Sternik, 2002), more severe lesions prevailed significantly at the convexity in previous morphological studies (Cotrufo, 2001; Agozzino, 2002). Asymmetric expansion seems to be even more pronounced in the setting of ascending dilations associated with congenital BAV. In spite of the recent increasing interest in ultrastructural changes underlying medial degeneration of the aortic dilations, often associated with Bicuspid Aortic Valve (BAV), its pathogenesis remains undefined. Some authors have claimed that the aortic wall pathology could be mainly secondary to abnormal post-valvular hemodynamics. Robicsek has highlighted the eccentricity of blood flow in the ascending aorta downstream to a BAV with consequent uneven wall stress distribution (Palmieri, 2001; Robicsek, 2004). Since flow characteristics are argued to possibly influence morphological and even ultrastructural vessel wall abnormalities (Stehbens, 1996), this typical asymmetric stress pattern could account for the asymmetric expansion of BAV-associated aneurysms. However, other Authors maintain that aortic wall weakness in BAV patients could be genetically determined, rather than conditioned by flow disturbances. Recently, an abnormality in the process of matrix microfibrillar component remodeling (inadequate synthesis, degradation and/or transport) has been advocated as a mechanism involved in the development of aortic dilations with BAV (Fedak, 2003). To our knowledge, only one study investigated the content of medial matrix constituents other than fibrillin and/or elastin by immunohistochemistry (Nataatmadja, 2003). As it is known that ECM is a dynamic and highly specialized structure, involved also in signal transduction pathways, we studied the distribution of its major components, such as type I and III Collagen, Fibronectin and Tenascin, as well as of Laminins and type IV Collagen, which are also directly involved in Basal Lamina composition, in order to determine which alterations are related to the aortic wall dilation.

In order to investigate on the asymmetric progression of aorta dilations we studied the distribution of the ECM proteins both in aortic convexity and concavity in our patients.

\section{Materials and Methods}

\section{Patients}

27 patients with congenital bicuspid aortic valve referred for surgical treatment of proximal aorta dilation constituted the present study sample (17 males, 10 females; mean age $56.3 \pm 9.2$ years). The investigation conforms with principles outlined in the Declaration of Helsinki (Cardiovascular Research, 1997; 35:2-4)

For comparison, normal aortic wall specimens were retrieved during multiorgan harvesting from 10 heart donors (mean age $33 \pm 8$ years) with no evidence for aneurysmal or atherosclerotic disease of the aorta nor BAV.

At surgery, two samples of aortic wall were harvested from each patient distally to the sinotubular junction at the aortic convexity and concavity. Each specimen was then sectioned into three parts: one of them was used for Western Blot analysis, one for PCR and molecular biology and the last one was paraffin embedded for immunohistochemistry.

We compared the convexity of each aortic sample with the concavity of the same vessel and both of them with the respective control samples, and focused our attention on BL composition and organization. 


\section{Western Blot}

Samples were washed in PBS and then incubated with lysis buffer (RIPA buffer), containing protease inhibitors, for $30^{\prime}$ at $4^{\circ} \mathrm{C}$. Lysates were centrifuged at $14,000 \mathrm{rpm}$ for $10^{\prime}$ at $4^{\circ} \mathrm{C}$; protein concentration was determined by Protein assay (BioRad, Richmond, C0). For each sample, the same amount of total proteins was added to Laemmli sample buffer and separated by electrophoresis in $6 \%$ SDS-polyacrylamide gel. Gels were then electroblotted on PVDF filters (Millipore, Bedford, MA); membranes were blocked in 5\% fat-free dry milk for 1 hour at room temperature and then incubated overnight at $4^{\circ} \mathrm{C}$ with antibodies against Laminin chains $\alpha 2, \beta 1$ and $\beta 2$ (LM), Tenascin (TN), type I, type III and type IV Collagen (Sigma, St.Louis, Missouri, USA) or with anti-Fibronectin (Chemicon Int., Temecula, CA, USA). After washing, the membranes were incubated for $\mathrm{lh}$ at room temperature with horseradish peroxidase-conjugated goat secondary antibodies (BioRad).

After further washing, membranes were developed in Luminol substrate and exposed to film (ECL for Laminin and Fibronectin, Amersham Biosciences, UK and ECLplus for Tenascin, Pierce, USA). Computer-acquired images were quantified using ImageQuant software (Amersham Biosciences, UK).

\section{Semiquantitative RT-PCR}

RT-PCR was used to analyze target gene expression in the present study. Total RNA was isolated by

Table 1. Oligonucleotides sequences used for semiquantitative RT-PCR.

\begin{tabular}{|c|c|c|c|}
\hline Target mRNA & Primer & Sequence & PCR product (bp) \\
\hline \multirow[t]{2}{*}{ GAPDH } & Forward & 5'GAGTCAACGGATTTGGTC3' & 238 \\
\hline & Reverse & ${ }^{5}$ 'TGATTTIGGAGGGATCT3' & \\
\hline \multirow[t]{2}{*}{ Fibronectin } & Forward & '5CCAACTGGCATTGACTाTा' & 136 \\
\hline & Reverse & '5TCGAGGTCTTTCACTGAAG3' & \\
\hline \multirow[t]{2}{*}{ Laminin $\alpha-2$} & Forward & '5AAAGTATCTGTGTCTTCAGGAGGTGC3' & 474 \\
\hline & Reverse & '5'GCAGCCAGTGAATGTAATCACACGTC3' & \\
\hline \multirow[t]{2}{*}{ Collagen I $\alpha-1$} & Forward & '5TCCCCAGAAGACACAGGAA3' & 203 \\
\hline & Reverse & 'TCAAAAACGAAGGGGAGATG3' & \\
\hline \multirow[t]{2}{*}{ Collagen IV $\alpha-2$} & Forward & '5CCAAGGAAGAGGTGGTGT3' & 169 \\
\hline & Reverse & '5'GTGCTTCACCAGGAGGTA3' & \\
\hline \multirow[t]{2}{*}{ Tenascin-X } & Forward & 5'CCTTGTCCTCCACACCAA3' & 227 \\
\hline & Reverse & 5'GGATCTCCTGGTTCTGTC3' & \\
\hline
\end{tabular}

lysing the frozen aortic vessel tissue samples (150$300 \mathrm{mg}$ ) in Trizol solution (Life Technologies, GIBCO BRL) according to the supplier's protocol. RNA was precipitated and quantified by spectroscopy. $2 \mathrm{mg}$ of total RNA of each sample was reversely transcribed using the First - Strand cDNA Synthesis Kit (Amersham Pharmacia Biotech) according to the protocol supplied by the manufacturer. The random hexamer primers provided in the kit were used. The same cDNA product obtained from each sample was used for subsequent PCR amplification with the primer sets prepared for the target gene and GAPDH housekeeping gene. The amplification of the GAPDH gene was used as double internal control. The ratio between the samples and the housekeeping gene was calculated to normalize for initial variations in sample concentration and as a control for reaction efficiency. Primer sequences were designed using the software Primer 3 (developed by Steve Rozen, Helen J Skaletsky) available on-line at http://wwwgenome.wi.mit.edu (Table 1). Semiquantitative Polymerase Chain Reaction (PCR) was performed using the following conditions: $95^{\circ} \mathrm{C}, 5 \mathrm{~min}$ initial denaturing phase; $95^{\circ} \mathrm{C}, 1 \mathrm{~min} ; 55^{\circ} \mathrm{C}, 1 \mathrm{~min} ; 72^{\circ} \mathrm{C}$, 1 min for 35 cycles; $72^{\circ} \mathrm{C}, 10 \mathrm{~min}$, final extension. The reaction was carried out in a total volume of $50 \mathrm{~mL}$ containing $3 \mu \mathrm{L}$ of $\mathrm{cDNA}, 10-20 \mathrm{pmol}$ of each primer, $200 \mathrm{mM}$ each of dNTP, $1.5 \mathrm{mM} \mathrm{MgCl}$ and 1 unit of Taq polymerase with the reaction buffer supplied with the kit. In each experiment, possible DNA contamination was determined by a control reaction in which cDNA was omitted from the reaction mixture and replaced by DNAse and RNAse free water. The amplified products ( $12 \mu \mathrm{l}$ of each sample) were analyzed by electrophoresis in a $2 \%$ agarose gel containing ethidium bromide, followed by photography under ultraviolet illumination. The levels of Extracellular Matrix proteins mRNA were estimated by densitometric scanning and normalized against GAPDH loading controls. Densitometric analyses of the PCR products were performed using the ImageJ v1.29 software (developed by Wayne Rasband) available on-line at http://rsb.info.nih.gov/ij/. All PCR products were purified using QIAquick PCR purification kit (Qiagen, Santa Clarita, CA, USA) and their identities verified by automated DNA forward and reverse sequencing using a dideoxy terminator reaction chemistry for sequence analysis on the Applied biosystem Model 373A DNA sequencer. 

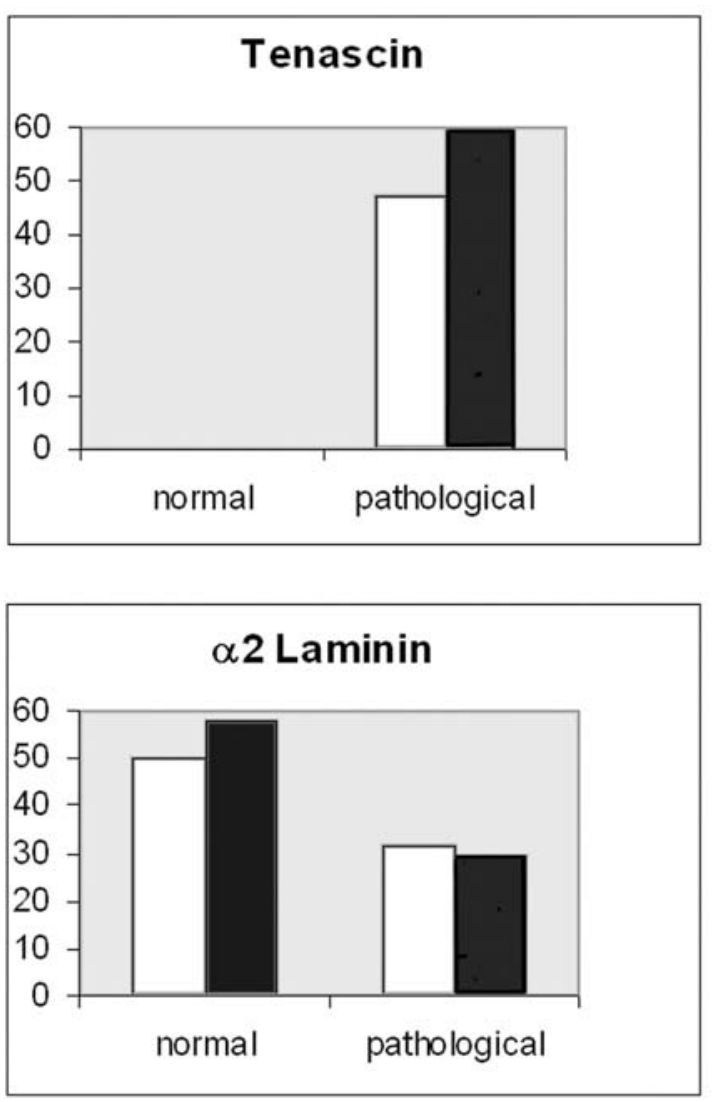
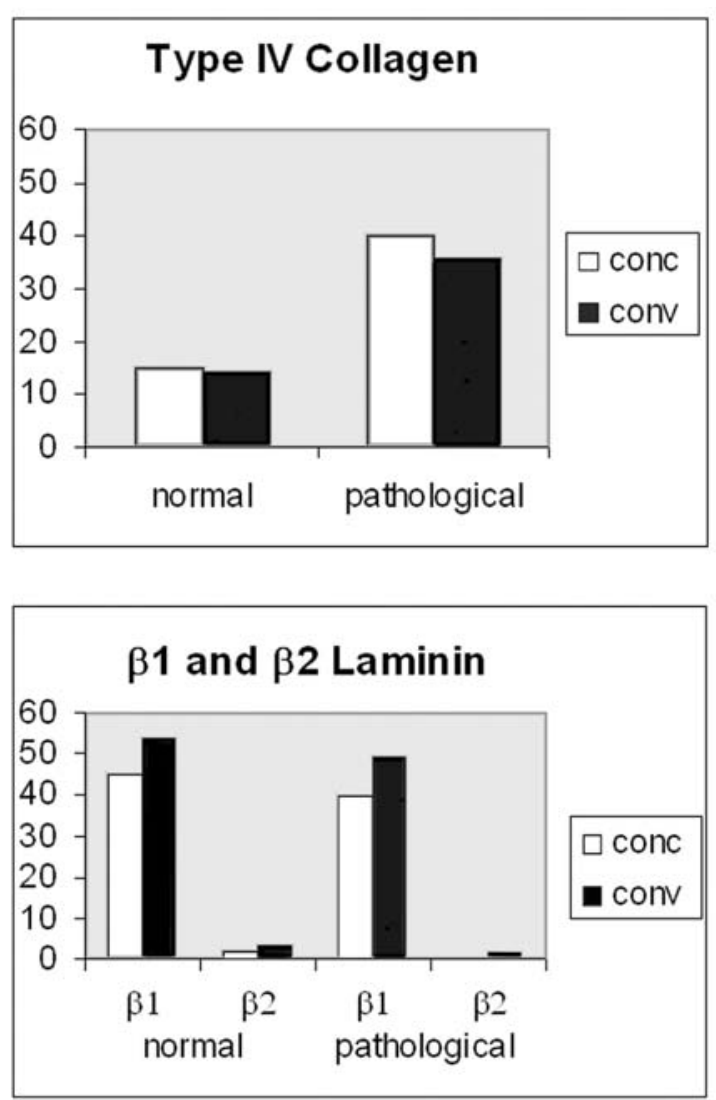

Figure 1. Western Blot shows a significant increase of Collagen IV and Tenascin in pathological samples. As regards Laminin chains, $\alpha 2$ significantly decreases in dilated aortas, $\beta 1$ is abundant in all specimens and only slightly decreases in dilated aortas and $\beta 2$ is poorly expressed in controls and is virtually absent in dilated samples.

\section{Immunohistochemistry}

Specimens were fixed in buffered 10\% Formalin, embedded in paraffin and sectioned. Serial $4 \mu$ thick sections of aortic specimens were deparaffinized, covered with primary monoclonal antibodies against types I and IV Collagen, Tenascin and Laminin or polyclonal antibody for Fibronectin (Sigma, St.Louis, Missouri, USA) and type III Collagen (Santa Cruz Biotechnology, INC, Santa Cruz, CA, USA) and incubated in a moist chamber for 1 hour at $37^{\circ} \mathrm{C}$. After washings in PBS, the sections were covered with fluoresceinated secondary antibodies (Sigma, St.Louis, Missouri, USA) and incubated again in the same conditions.

After the staining of the nuclei with Propidium Iodide, the sections were mounted in Vectashield and observed with Leica DMLB fluorescence microscope and with Zeiss LSM510 confocal microscope. Every sample was evaluated by three independent observers, using a four point arbitrary scale ranging from 0 to 3 .

\section{Statistical analysis}

The results are reported as mean values +/- SD. Student's t-test was performed in each experiment to evaluate the modifications observed in comparison with the normal aortic walls. Differences at $p<0.05$ were considered statistically significant.

\section{Results}

\section{Western Blot}

WB results show a decreased production of type I and type III Collagen in the pathological specimens when compared with normal aortic wall; in addition, there is no significant difference between convexity and concavity (data not shown).

Collagen IV and Tenascin are increased in pathological samples. The small amount of Tenascin required the detection of femtomoles of total protein, while all other proteins were expressed in nanomoles (Figure 1 ).

Fibronectin is only slightly more abundant in 
healthy subjects than in pathological samples; without any significant difference between concavity and convexity (data not shown).

Laminin chains $\beta 1$ and $\beta 2$ amounts differ strongly, as $\beta 2$ is poorly expressed in all specimens and is totally absent in dilated concavity, while $\beta 1$ amount is high in all specimens and only slightly decreases in pathological aortas; Laminin $\alpha 2$ chain expression significantly decreases in pathological aortas (Figure 1).

\section{Semiquantitative RT-PCR}

RT-PCR provided data on the mRNA expression of ECM proteins studied.

As regards Fibronectin, the great variability among patients is confirmed also by mRNA expression, with a slight decrease in all pathologic aortas when compared with normal ones (data not shown). The level of mRNA for Laminin $\alpha 2$-chain is always decreased both in concavity and in convexity from pathological samples when compared with normal ones. This decrease is statistically significant (Figure 2).

The level of mRNA for type X Tenascin, the only type we were able to demonstrate in our specimens, is clearly increased in pathological specimens when compared with normal ones. This increase is more evident in aortic walls convexity than in concavity (Figure 2).

Type I Collagen mRNA is slightly decreased in pathologic samples when compared with controls (data not shown) while type IV Collagen mRNA is more expressed in pathological aortic walls than in healthy subjects, and more in concavity than in convexity (Figure 2 ).

\section{Immunohistochemistry}

Our morphological observation of Extra Cellular Matrix of the aortic tissue demonstrated important changes in the tridimensional arrangement of the major components of the wall more clearly than evaluation of the expression of the ECM proteins. The thickness of the normal aortic wall, when measured on histological sections, was $1.82 \pm 0.05$ $\mathrm{mm}$ in concavity and $1.92 \pm 0.05 \mathrm{~mm}$ in convexity in controls. Pathologic specimens are always thinner, with wall thickness of $1.18 \pm 0.04 \mathrm{~mm}$ and $1.13 \pm 0.06 \mathrm{~mm}$, respectively.

The loss of organization of the fiber mesh in the media is evident in the distribution of all the components, but also the intimal layer showed some
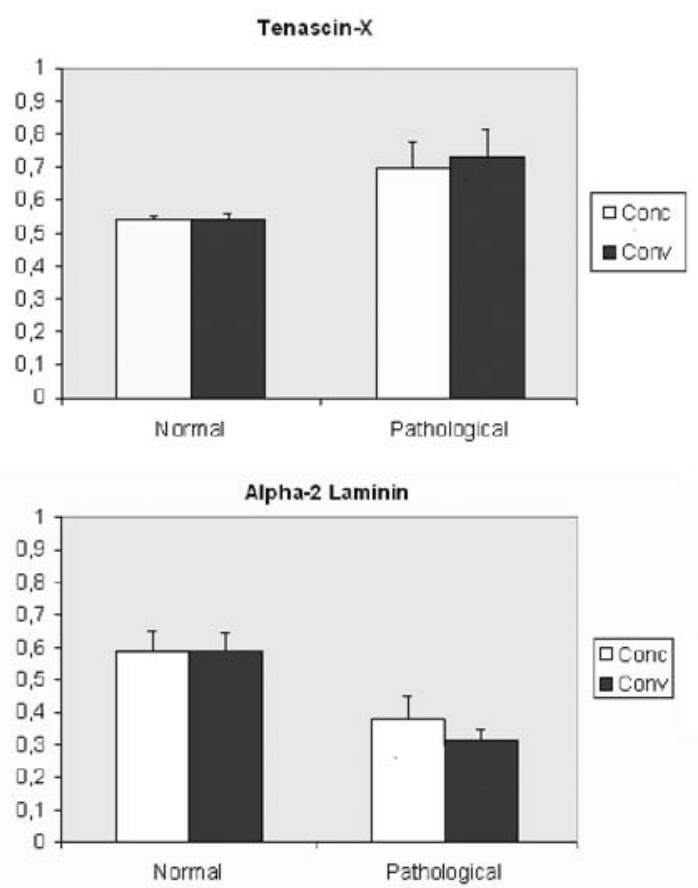

Figure 2. RT-PCR data demonstrate that the level of mRNA for Type $X$ Tenascin is clearly increased in pathological specimens when compared with normal ones. The level of mRNA for Laminin $\alpha 2$-chain is always decreased both in concavity and in convexity from pathological samples when compared with normal ones. This decrease is statistically significant.

important alterations. Our observations by confocal immunofluorescence are summarized in Table 2.

In control subjects, the expression and the distribution of ECM proteins were meanly uniform between convexity and concavity. Fibronectin and Laminin were abundant in the intimal and the outer media layers; type III Collagen showed an irregular distribution in both aortic wall portions, type IV Collagen was virtually absent at the immunohistochemical observation. Type I Collagen was abundant, as expected.

Patients specimens show that types I and III Collagen have the same distribution in concavity and convexity and are less expressed when compared with controls. Type IV Collagen is more abun-

Table 2. Immunohistochemical evaluation of aortic wall.

\begin{tabular}{lcccccc}
\hline \hline & Col & Colll & ColV & Fibronectin & Tenascin & Laminin \\
\hline Normal Convexity & ++ & + & +- & + & +- & $+/++$ \\
Normal Concavity & ++ & $+/++$ & +- & + & +- & ++ \\
Dilated Convexity & +- & +- & + & ++ & +++ & + \\
Dilated Concavity & +- & +- & +++ & + & ++ & + \\
\hline
\end{tabular}



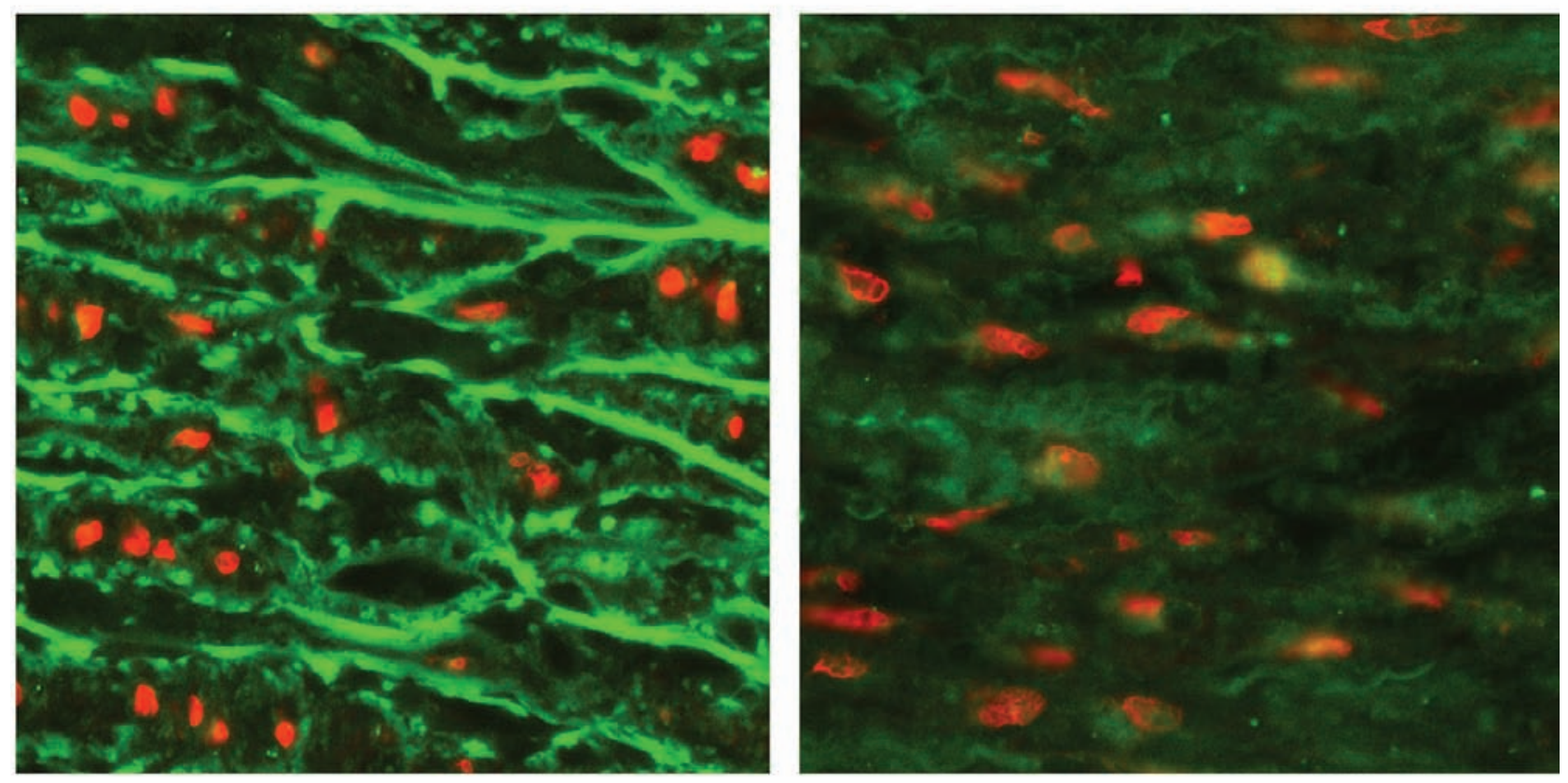

Figure 3. Laminin immunopositivity in normal aorta is on the left. The decrease of Laminin in dilated aortas is clearly demonstrated by the image on the right. Confocal microscopy, 600X.
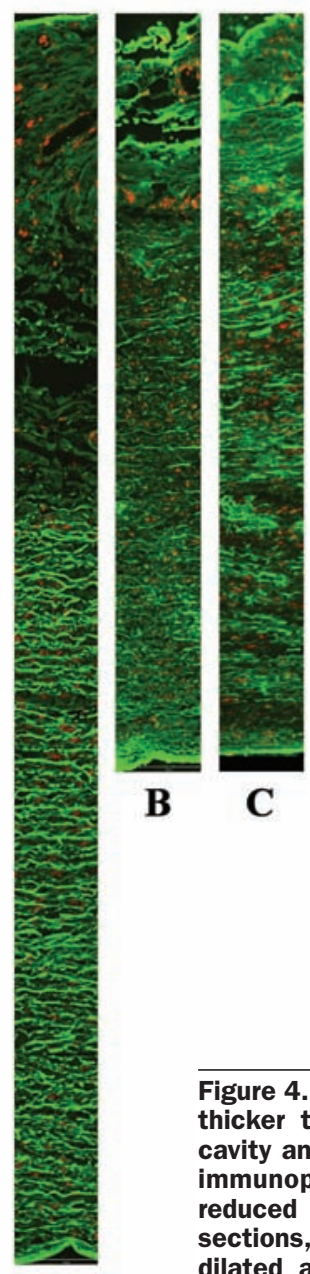

B
C

Figure 4. The normal aortic wall $(A)$ is always thicker than pathologic specimens (B concavity and $C$ convexity of dilated aorta). The immunopositivity for Tenascin is quite reduced in the initial layer of all the normal sections, and it increases in comvexity of dilated aortic walls. Confocal micrographs; the bar corresponds to $100 \mu$. dant in pathological aortas, in particular in vessel concavity. Laminin decreases in dilated aortas (Figure 3). Fibronectin amount is only slightly increased in dilated convexity. Generally, the immunopositivity for Tenascin is reduced in the intimal layer of all the normal sections examined, but it increases in dilated aortic walls, particularly in the convexity (Figure 4).

All the observed alterations in ECM proteins distribution affected mainly the media layer, but individual modifications of the normal ECM mesh of the other layers were evident in some cases.

\section{Discussion}

To the best of our knowledge this is the first morpho-biomolecular study investigating the pattern of distribution and expression of the major components of Extra Cellular Matrix in aortic media lesions in patients with asymmetrically dilated aorta. Basal lamina of the dilated aortic wall presented important changes in the tridimensional arrangement of its major components. All the examined vessels showed a thinner wall when compared with normal samples. Our data were consistent with the common macroscopic observation that ascending aorta dilations tend to expand asymmetrically, with prevalent involvement of the vessel 
Table 3. Laminins isoforms composition.

\begin{tabular}{cc}
\hline \hline Laminin isoforms & Chains \\
\hline Laminin-2 & $\alpha 2 \beta 1 \gamma 1$ \\
Laminin-3 & $\alpha 1 \beta 2 g \gamma$ \\
Laminin-4 & $\alpha 2 \beta 2 g \gamma$ \\
Laminin-8 & $\alpha 4 \beta 1 g \gamma$ \\
Laminin-9 & $\alpha 4 \beta 2 g \gamma$ \\
Laminin-10 & $\alpha 5 \beta 1 g \gamma$ \\
Laminin-11 & $\alpha 5 \beta 2 g \gamma$ \\
\hline
\end{tabular}

convexity. In our study, statistically significant differences in the amount and the distribution of ECM proteins are demonstrated in particular for Laminin $\alpha 2$ chain, Tenascin and type IV Collagen.

PCR and Western Blot revealed a disomogeneous distribution of Laminin chains $\alpha 2, \beta 1$ and $\beta 2$ in concavity and convexity of both control and dilated aortic wall. $\beta 1$ chain is well represented in all specimens while $\beta 2$ is always poorly expressed and totally absent in dilated concavity. mRNA for $\alpha 2$ chain is always decreased both in concavity and in convexity from pathological samples when compared with normal ones. These observations suggest that Laminins 8 and 10 could be the isoforms of Laminins prevailing in dilated aortas among those more usually present in blood vessels.

In fact, Laminins 4 and 2 contain $\alpha 2$ chain which decreased in all pathological samples; Laminins 3, 9 and 11 contain $\beta 2$ chain which is virtually absent in our specimens (Table 3 ).

The amount of type IV Collagen in dilated vessels is strongly increased in comparison with normal ones and its distribution, which was uniform in normal aortic wall, becomes irregular in pathological specimens.

The significant differences in the amount and the distribution of type IV Collagen, Tenascin and Laminins are evident between concavity and convexity of the same aortic sample as well as in comparison with normal subjects. In addition, the structural arrangement of type I Collagen, which is considered the mechanical scaffold of the vessel, is strongly altered and meanly disomogeneous in the whole extension of the media layer, also if its amounts do not show strong differences. Biomolecular data generally confirm morphological observations, with the only exception of Fibronectin; this slight difference is not significant and is due to the necessity of using different antibodies for the two procedures.

The synthesis of ECM components may vary, as demonstrated by the reduced amount of Tenascin in normal aortas and by the increase in the synthesis of this protein in dilated vessels, in particular in the convexity. From this point of view, the molecular biology shows little amount of Tenascin, which are of course undetectable with the morphological techniques. The thickness of the aortic pathological walls varies as well, with a constant decrease in dilated vessels when compared to normal ones. The complexity of cell-matrix interactions prevent so far from an exhaustive interpretation of the significance of protein abnormalities in aortic aneurysms. Further information will be acquired in the next future by comparing dilated aortic wall in patients with BAV with the more symmetrical aneurysms in patients with tricuspid aortic valve disease, as well as by comparing between BAV aortas with or without dilation. Nevertheless, our data, i.e. the asymmetric pattern of expression of some ECM and BL components, support the hypothesis that some causative relation exists between the flow disturbances due to the eccentrically directed blood jet from the BAV and the development of aortic dilation. Aortic wall muscle cells are known (Bardy 1996, Mackie 1992) to react to wall stress by changing phenotype and producing both ECM components (such as type IV Collagen and Tenascin) and enzymes that eventually degrade the normal structural constituents (such as Elastin and Collagens). It is difficult, so far, to understand why the individual molecules of the basal lamina are affected by different alterations: for unknown reasons, the mechanism of protein overproduction seems to prevail for some proteins (e.g. Tenascin), while over-degradation or under-expression for others (e.g. Laminin). Mechanical properties of aortic wall could be strongly modified by these remodeling phenomena, which probably induce a localized decrease in wall resistance to blood flow with consequent dilation, prevailing at the convexity where wall stress is greater. In particular, the significant increase of Tenascin could be a physiological reaction to the mechanical stress of the wall. Further investigations are needed to clarify the role of $\alpha 2$ and $\beta 1$ chains of Laminins in tissue remodeling; so far, Laminin 2 seems the candidate for the more significant change. The decrease of this isoform, also called merosin, plays an important role in many muscle pathologies characterized by weakness (Jones, 2001).

On the basis of these results, it becomes clear that 
if an intrinsic genetically mediated wall weakness exists, it may predispose the arterial wall to degeneration when locally subjected to high flow and shear stress. Conclusive evidences will be reached only when involved genes will be identified and their regulative mechanisms, including environmental influences, disclosed.

\section{Acknowledgments}

The Authors are very grateful to STORZ Medical ITALIA which supported their work.

\section{References}

Agozzino L, Ferraraccio F, Esposito S, et al. Medial degeneration does not involve uniformly the whole ascending aorta: morphological, biochemical and clinical correlations. Eur J Cardiothorac Surg 2002;21:675-82.

Bardy N, Merval R, Benessiano J, et al. Pressure and angiotensin II synergistically induce aortic fibronectin expression in organ culture model of rabbit aorta. Evidence for a pressure-induced tissue reninangiotensin system. Circ Res 1996;79:70-8.

Bauer M, Pasic M, Schaffarzyk R et al. Reduction aortoplasty for dilatation of the ascending aorta in patients with bicuspid aortic valve. Ann Thorac Surg. 2002;73:720-3; discussion 724.

Choo SJ, McRae G, Olomon JP, et al. Aortic root geometry: pattern of differences between leaflets and sinuses of Valsalva. J Heart Valve Dis 1999;8:407-15.

Cotrufo M, De Santo LS, Esposito S, et al. Asymmetric medial degeneration of the intrapericardial aorta in aortic valve disease. Int $J$ Cardiol 2001;81:37-41.

Dagum P, Green GR, Nistal FJ, et al. Deformational dynamics of the aortic root: modes and physiologic determinants. Circulation 1999;100:II54-62.

Dingemans KP, Teeling $P$, Lagendijk JH, et al. Extracellular matrix of the human aortic media: an ultrastructural histochemical and immunohistochemical study of the adult aortic media. Anat Rec 2000;258:1-14.
Fedak PW, de Sa MP, Verma S, et al. Vascular matrix remodeling in patients with bicuspid aortic valve malformations: implications for aortic dilatation. J Thorac Cardiovasc Surg 2003;126:797-806.

Grande KJ, Cochran RP, Reinhall PG, et al. Stress variations in the human aortic root and valve: the role of anatomic asymmetry. Ann Biomed Eng 1998;26:534-45.

Jones KJ, Morgan G, Johnston $H$, Tobias $V$, et al. The expanding phenotype of laminin a2 chain (merosin) abnormalities: case series and review. J Med Genet 2001; 38:649-57.

Lansac E, Lim HS, Shomura $Y$, et al. A four-dimensional study of the aortic root dynamics. Eur J Cardiothorac Surg 2002;22:497-503.

Mackie EJ, Scott-Burden T, Hahn AW, et al. Expression of tenascin by vascular smooth muscle cells. Alterations in hypertensive rats and stimulation by angiotensin II. Am J Pathol. 1992;141:377-88.

Miner JH and Yurchenco PD: Laminin Functions in tissue morphogenesis. Annu Rev Cell Dev Biol 2004;20:255-84.

Nataatmadja M, West M, West J, et al. Abnormal extracellular matrix protein transport associated with increased apoptosis of vascular smooth muscle cells in marfan syndrome and bicuspid aortic valve thoracic aortic aneurysm. Circulation 2003;108 Suppl 1:II329-34.

Palmieri V, Bella JN, Arnett DK, et al. Aortic root dilatation at sinuses of Valsalva and aortic regurgitation in hypertensive and normotensive subjects: the Hypertension Genetic Epidemiology Network Study. Hypertension 2001;37:1229-35.

Patarroyo M, Tryggvason K, Virtanen I. Laminin isoforms in tumor invasion, angiogenesis and metastasis. Cancer Biology 2002; 12:197-207

Robicsek F. Bicuspid versus tricuspid aortic valves. J Heart Valve Dis 2003; 12:52-3.

Robicsek F, Thubrikar MJ, Cook JW, Fowler B. The congenitally bicuspid aortic valve: how does it function? why does it fail? Ann Thorac Surg 2004; 77:177-85.

Schlatmann TJ, Becker AE. Histologic changes in the normal aging aorta: implications for dissecting aortic aneurysm. Am J Cardiol 1977;39:13-20.

Stehbens WE. Structural and architectural changes during arterial development and the role of hemodynamics. Acta Anat 1996;157:261-74.

Sternik L, Zehr KJ, Schaff HV. A method of repair for asymmetric aneurysmal dilatation of the ascending aorta. Ann Thorac Surg 2002;73:1332-4. 